\title{
Weak ergodicity breaking induced by global memory effects
}

\author{
Adrián A. Budini \\ Consejo Nacional de Investigaciones Científicas y Técnicas (CONICET), \\ Centro Atómico Bariloche, Avenida E. Bustillo Km 9.5, (8400) Bariloche, \\ Argentina, and Universidad Tecnológica Nacional (UTN-FRBA), \\ Fanny Newbery 111, (8400) Bariloche, Argentina
}

(Dated: September 3, 2018)

\begin{abstract}
We study the phenomenon of weak ergodicity breaking for a class of globally correlated random walk dynamics defined over a finite set of states. The persistence in a given state or the transition to another one depends on the whole previous temporal history of the system. A set of waiting time distributions, associated to each state, set the random times between consecutive steps. Their mean value is finite for all states. The probability density of time-averaged observables is obtained for different memory mechanisms. This statistical object explicitly shows departures between time and ensemble averages. While the mean residence time in each state may result divergent, we demonstrate that this condition is in general not necessary for breaking ergodicity. Hence, global memory effects are an alternative mechanism able to induce this property. Analytical and numerical calculations support these results.
\end{abstract}

PACS numbers: 05.70.Ln, 05.40.-a, 89.75.-k

\section{INTRODUCTION}

Ergodicity plays a fundamental role in the formulation of statistical physics. This property is usually stated by saying that ensemble average and time average of observables are equals, the last one being taken in the long time (infinite) limit. In contrast with thermodynamical systems, where the lack or ergodicity is induced by a spontaneous symmetry breaking [1], the disparity between ensemble and time averages may also be found as an emergent property of complex systems. Named as weak ergodicity breaking (EB) [2], this feature is induced by the power-law nature of the statistical distributions associated to the observables and their dynamics [2, 3].

Time averages in presence of weak EB remain random even in the long time limit. Their statistics, termed as weakly non-ergodic statistical physics [4, 5], define a still very active line of research. Continuous time random walk characterized by divergent trapping times is a natural frame where weak EB was studied [4 9]. In addition, diverse kinds of complex anomalous diffusion processes are a natural partner of weak EB. Analysis were performed for particles embedded in heterogenous media 10], periodic potentials 11, and in homogeneous disordered media [12]. Geometric 13], escaled 14] and ultraslow 15] Brownian motions, as well as diffusion induced by the combined action of different driven noises [16 18], convoluted memory processes [19] and Langevin dynamics [20] also were characterized from a similar perspective.

In addition to its theoretical interest, weak EB was also found in different physical systems such as deterministic dynamics 21 24] and blinking nanocrystals [25, 26]; also in molecular transport [27] and tracking of biological single molecules [28 32] such as lipid granules 31], and diffusion in the plasma membrane of living cells 32]. Weak EB also arises in complex networks [33, 34], fluid turbulence [35] and brain dynamics 36].
Weak EB can be studied in systems that have associated a stationary state, such as for example random walks on finite domains, and also in non-stationary systems such as unbounded diffusive ones (see for example Refs. [4] and [28] respectively). Independently of the dimensionality, weak EB is in general associated or related to some underlying self-similiar (effective) mechanism characterized by power-law distributions. The main goal of this paper is to demonstrate that systems whose dynamics involves global memory effects may also develop EB. Furthermore, we establishes that the lack of ergodicity may happens even in absence of statistical properties (residence times) characterized by dominant power-law distributions.

Global memory (or correlation) effects refer to systems whose stochastic dynamics at a given time depends on its whole previous temporal history (trajectory). These kinds of dynamics has been studied previously [37-45], mainly as a mechanism that induces superdiffusion. In contrast, here we study random walk processes defined over a finite set of states where the persistence in a given state or the transition to another one depends on the previous system trajectory. The random times between consecutive steps is defined by a set of waiting time distributions with finite average times. In addition, our main results rely on alternative memory mechanisms. They are related to a Pólya urn dynamics [46 50], which is one of the simplest models of contagion process, being of interest in various disciplines [47]. In contrast to other global correlation mechanisms, the urn-like dynamics is able to induce weak EB. Interestingly, the departure from ergodicity arises even when the (average) residence times in each state are finite.

The paper is organized as follows. In Sec. II, we introduce the globally correlated random walk model. The probability density of time-averaged observables is obtained in general. In Sec. III, we study three different 
global memory mechanisms: the elephant random walk model, a random walk driven by an urn-like dynamics, and an imperfect case of the last one. In Sec. IV, for all models, we obtain the probability density of the residence times. Sec. V is devoted to the Conclusions. Analytical calculations that support the main results are presented in the Appendixes.

\section{FINITE RANDOM WALK WITH GLOBAL MEMORY EFFECTS}

In this section we introduce the globally correlated random walk model and study its properties. The probability density of time-averaged observables is also obtained.

\section{A. Model}

The system is characterized by a finite set of states $\mu=1, \cdots L$. To each state $\mu$ we assign a waiting time distribution $w_{\mu}(t)$, which gives the statistics of times between consecutive steps of the stochastic dynamics. We assume that all average times

$$
\tau_{\mu} \equiv \int_{0}^{\infty} d t w_{\mu}(t) t
$$

are finite, $\tau_{\mu}<\infty$.

The stochastic dynamics is as follows. At the beginning (initial time), each state is selected in agreement with a set of probabilities $\left\{p_{\mu}\right\}_{\mu=1}^{L}, 0 \leq p_{\mu} \leq 1$, normalized as $\sum_{\mu=1}^{L} p_{\mu}=1$. Given that a state $\mu$ is selected, the system remains in it during a random time selected in agreement with the waiting time distribution $w_{\mu}(t)$. After this step, the system may remain in the same state or jump to another one. Hence, it may persists in the same state, remaining an extra time interval chosen in agreement with the same waiting time distribution, or jump to a different state with a different waiting time distribution. This dynamic repeats itself in time after each step, where step refers to the process of selecting the next state.

The state corresponding to the next step is chosen in agreement with a conditional probability $\mathcal{T}_{n}\left(\left\{n_{1}, n_{2} \cdots n_{L}\right\} \mid \mu\right)$ [denoted as $\left.\mathcal{T}_{n}\left(\left\{n_{\nu}\right\} \mid \mu\right)\right]$. Here, $n$ indicates the number of steps performed up to the present time, while $n_{\nu}$ gives the number of times that each state $\nu$ was chosen previously. Then, $n=\sum_{\nu=1}^{L} n_{\nu}$. The dependence of the process on the whole previous trajectory (global correlation) is given by the dependence of $\mathcal{T}_{n}\left(\left\{n_{\nu}\right\} \mid \mu\right)$ on the set $\left\{n_{\nu}\right\}_{\nu=1}^{L}$. The previous definitions completely characterize the stochastic dynamics in terms of the initial probabilities $\left\{p_{\mu}\right\}_{\mu=1}^{L}$, the waiting time distributions $\left\{w_{\mu}(t)\right\}_{\mu=1}^{L}$ and the conditional (or transition) probabilities $\mathcal{T}_{n}\left(\left\{n_{\nu}\right\} \mid \mu\right)$.

For the studied models [see Eqs. (15), (16), and (21)], as a consequence of the memory effects, the following property is observed. In the long time limit $(t \rightarrow \infty)$, which also correspond to a divergent number of steps $(n \rightarrow \infty)$, the fractions

$$
f_{\mu}=\lim _{n \rightarrow \infty} \frac{n_{\mu}}{n}
$$

$\sum_{\mu=1}^{L} f_{\mu}=1$, may become random variables whose values depend on each particular realization. Their probability density is denoted by $\mathcal{P}\left(\left\{f_{\mu}\right\}\right)$, which satisfies the normalization condition $\int_{\Lambda} d f_{1} \cdots d f_{L-1} \mathcal{P}\left(\left\{f_{\mu}\right\}\right)=1$. Here, $\Lambda$ is the region defined by the condition $\sum_{\nu=1}^{L} f_{\mu}=$ 1 . The average of $f_{\mu}$ over an ensemble realizations, denoted by $\langle\cdots\rangle$, is

$$
\left\langle f_{\mu}\right\rangle=\int_{\Lambda} d f_{1} \cdots d f_{L-1} f_{\mu} \mathcal{P}\left(\left\{f_{\nu}\right\}\right)
$$

At a given time $t$, with $P_{\mu}(t)$ we denote the (ensemble) probability $\left[\sum_{\mu=1}^{L} P_{\mu}(t)=1\right]$ that the system is in the (arbitrary) state $\mu$. This object is characterized in Appendix A from the dynamics defined previously. The stationary probability reads $P_{\mu}^{\text {st }} \equiv \lim _{t \rightarrow \infty} P_{\mu}(t)$. It can be written in terms of $\mathcal{P}\left(\left\{f_{\nu}\right\}\right)$ as

$$
P_{\mu}^{\mathrm{st}}=\left\langle\frac{f_{\mu} \tau_{\mu}}{\sum_{\mu^{\prime}=1}^{L} f_{\mu^{\prime}} \tau_{\mu^{\prime}}}\right\rangle,
$$

where $\tau_{\mu}$ is defined by Eq. (1). In Appendix A we also derive this result. Basically it say us that in each realization the system reaches a (random) stationary state defined by the weights $\left(f_{\mu} \tau_{\mu}\right) / \sum_{\mu^{\prime}=1}^{L} f_{\mu^{\prime}} \tau_{\mu^{\prime}}$. In consequence, $P_{\mu}^{\text {st }}$ depends on which memory mechanism drives the stochastic dynamics.

\section{B. Time-averaged observables}

To each state $\mu$, we assign an observable with value $\mathcal{O}_{\mu}$. Hence, each realization of the random walk defines a corresponding trajectory $\mathcal{O}(t)$. In the stationary regime, its ensemble average $\langle\mathcal{O}\rangle_{\text {st }} \equiv \lim _{t \rightarrow \infty}\langle\mathcal{O}(t)\rangle=$ $\lim _{t \rightarrow \infty} \sum_{\mu=1}^{L} P_{\mu}(t) \mathcal{O}_{\mu}$, is

$$
\langle\mathcal{O}\rangle_{\mathrm{st}}=\sum_{\mu=1}^{L} P_{\mu}^{\mathrm{st}} \mathcal{O}_{\mu}
$$

where the weights follows from Eq. (4). On the other hand, its time average is defined as $\mathcal{O} \equiv$ $\lim _{t \rightarrow \infty}(1 / t) \int_{0}^{t} d t^{\prime} \mathcal{O}\left(t^{\prime}\right)$, which leads to

$$
\mathcal{O}=\lim _{t \rightarrow \infty} \sum_{\mu=1}^{L}\left(\frac{t_{u}}{t}\right) \mathcal{O}_{\mu} .
$$

Here, $t_{u}$ is the total residence time in the state $\mu$ in the interval $(0, t)$. Hence, $\sum_{\mu=1}^{L} t_{u}=t$.

Even when a long time limit is present in the previous definition, the observable $\mathcal{O}$ may be a random object that 
depends on each particular realization. Its probability density can be written as

$$
P(\mathcal{O})=\lim _{t \rightarrow \infty}\left\langle\delta\left(\mathcal{O}-\sum_{\mu=1}^{L} \frac{t_{u}}{t} \mathcal{O}_{\mu}\right)\right\rangle,
$$

where, as before, $\langle\cdots\rangle$ denotes average over an ensemble of realizations and $\delta(x)$ is the Dirac delta function. Now, our goal is to calculate this object for the dynamics defined previously.

Given that the waiting time distributions are characterized by a finite average time $\tau_{\mu}$, Eq. (1), after invoking the law of large numbers, in the long time limit the total residence time $t_{u}$ in each state can be approximated as $t_{u} \simeq n_{\mu} \tau_{\mu}$. Consistently, the present time is $t \simeq \sum_{\mu=1}^{L} n_{\mu} \tau_{\mu}$. Hence, we can write

$$
\lim _{t \rightarrow \infty} \frac{t_{u}}{t} \simeq \lim _{n \rightarrow \infty} \frac{n_{\mu} \tau_{\mu}}{\sum_{\mu^{\prime}=1}^{L} n_{\mu^{\prime}} \tau_{\mu^{\prime}}}=\frac{f_{\mu} \tau_{\mu}}{\sum_{\mu^{\prime}=1}^{L} f_{\mu^{\prime}} \tau_{\mu^{\prime}}}
$$

where the last relation follows from Eq. (2). Taking into account that the fractions $\left\{f_{\mu}\right\}_{\mu=1}^{L}$ are characterized by the distribution $\mathcal{P}\left(\left\{f_{\mu}\right\}\right)$, Eq. (77) becomes

$$
\begin{aligned}
P(\mathcal{O})= & \int_{\Lambda} d f_{1} \cdots d f_{L-1} \mathcal{P}\left(\left\{f_{\mu}\right\}\right) \\
& \times \delta\left(\mathcal{O}-\sum_{\mu=1}^{L} \frac{f_{\mu} \tau_{\mu}}{\sum_{\mu^{\prime}=1}^{L} f_{\mu^{\prime}} \tau_{\mu^{\prime}}} \mathcal{O}_{\mu}\right)
\end{aligned}
$$

Therefore, $P(\mathcal{O})$ can be completely characterized after knowing the distribution $\mathcal{P}\left(\left\{f_{\mu}\right\}\right)$. Notice that the specific structure of the waiting time distributions $\left\{w_{\mu}(t)\right\}_{\mu=1}^{L}$ only appears through the average times $\left\{\tau_{\mu}\right\}_{\mu=1}^{L}$, Eq. (11).

\section{Ergodicity and localization}

For an ergodic dynamics the fractions $f_{\mu}$ [Eq. (2)] must be characterized by their ensemble average, Eq. (3). Hence,

$$
\mathcal{P}\left(\left\{f_{\mu}\right\}\right)=\delta\left(f_{1}-\left\langle f_{1}\right\rangle\right) \delta\left(f_{2}-\left\langle f_{2}\right\rangle\right) \cdots \delta\left(f_{L}-\left\langle f_{L}\right\rangle\right) .
$$

Inserting this expression into Eq. (9), it follows the distribution

$$
P(\mathcal{O})=\delta\left(\mathcal{O}-\langle\mathcal{O}\rangle_{\mathrm{st}}\right)
$$

where $\langle\mathcal{O}\rangle_{\text {st }}$ is given by Eq. (5) with the weights

$$
P_{\mu}^{\mathrm{st}}=\frac{\left\langle f_{\mu}\right\rangle \tau_{\mu}}{\sum_{\mu^{\prime}=1}^{L}\left\langle f_{\mu^{\prime}}\right\rangle \tau_{\mu^{\prime}}} .
$$

From Eqs. (4) and (10), we note that these weights correspond to the stationary probabilities of each state $\mu$ in the ergodic case. Hence, time averages and ensemble averages do in fact coincide.
The maximal departure with respect to ergodicity happens when the dynamics localize, that is, the system remains in the initial condition. This case corresponds to

$$
\mathcal{P}\left(\left\{f_{\mu}\right\}\right)=\sum_{\mu=1}^{L} p_{\mu} \delta\left(f_{1}\right) \cdots \delta\left(f_{\mu}-1\right) \cdots \delta\left(f_{L}\right) .
$$

Hence, Eq. (9) becomes

$$
P(\mathcal{O})=\sum_{\mu=1}^{L} p_{\mu} \delta\left(\mathcal{O}-\mathcal{O}_{\mu}\right)
$$

These limits are reached by the following memory mechanisms.

\section{EXAMPLES}

In the examples worked below, the stochastic dynamics may reach both the ergodic and localized regimes Eqs. (11) and (14) respectively. The distribution $\mathcal{P}\left(\left\{f_{\mu}\right\}\right)$ can be explicitly calculated and then the non-ergodic properties characterized through Eq. (9).

\section{A. Elephant random walk model}

This correlation model has been studied extensively in the recent literature as a mechanism for inducing superdiffusion [37-39]. In the present context, it is defined by the transition probability

$$
\mathcal{T}_{n}\left(\left\{n_{\nu}\right\} \mid \mu\right)=\varepsilon q_{\mu}+(1-\varepsilon) \frac{n_{\mu}}{n} .
$$

The positive weights $0<q_{\mu}<1$ are extra parameters normalized as $\sum_{\mu=1}^{L} q_{\mu}=1$. The parameter $\varepsilon$ assumes values in the interval $[0,1]$. The stochastic dynamics can be read as follows. With probability $\varepsilon$, and independently of the previous history, the new state is chosen in agreement with the probabilities $\left\{q_{\mu}\right\}_{\mu=1}^{L}$. On the other hand, with probability $(1-\varepsilon)$ each state is chosen in agreement with the weights $\left\{n_{\mu} / n\right\}_{\mu=1}^{L}$, which in fact depend on the whole previous history of the process.

For $\varepsilon=1$, the selection of the new state is completely random and independent of the previous history. Therefore, the system is ergodic in this case, Eq. (11). On the other hand, for $\varepsilon=0$ the dynamics localize, that is, the system remains in the initial condition, Eq. (14).

Even when the dynamics reaches the ergodic and localized regime, for intermediates values $0<\varepsilon<1$ the dynamics is ergodic. This property is demonstrated in Appendix $\mathrm{B}$. In fact, the distribution $\mathcal{P}\left(\left\{f_{\mu}\right\}\right)$ is delta distributed, Eq. (10), with $\left\langle f_{\mu}\right\rangle=q_{\mu}$.

\section{B. Random walk driven by an urn-like dynamics}

In the Pólya urn dynamics [46, 47] (initially) an urn contains many balls that, for example, are characterized 
by $L$ different possible colors. At each step, one determine the color of one ball taken at random and put into the urn one extra ball of the same color. A similar process can be defined by starting the urn with only one ball [48-50] (Blackwell-MacQueen urn). Its dynamics is defined by the following conditional probability, which is taken as the driving memory mechanism.

For the random walk over the $\mu=1, \cdots L$ states, we take the conditional probability [48, 49]

$$
\mathcal{T}_{n}\left(\left\{n_{\nu}\right\} \mid \mu\right)=\frac{\lambda q_{\mu}+n_{\mu}}{n+\lambda} .
$$

As before, the set of parameters $\left\{q_{\mu}\right\}_{\mu=1}^{L}$ is normalized to one. Instead, $\lambda$ is a positive free parameter. For $\lambda \rightarrow$ $\infty$ the dynamics loses any dependence on the previous history achieving in consequence an ergodic regime, Eq. (11). On the other hand, for $\lambda=0$, a localized regime is achieved, Eq. (14). Hence, the intermediate values of $\lambda$ avoid this regime and in consequence one can define a nontrivial dynamics starting from $n=1$.

For arbitrary values of $\lambda$, the probability density of the (asymptotic) fractions (2) is derived in Appendix C] It can be written as

$$
\mathcal{P}\left(\left\{f_{\mu}\right\}\right)=\left\{\sum_{\nu=1}^{L} \frac{p_{\nu}}{q_{\nu}} f_{\nu}\right\} D\left(\left\{f_{\mu}\right\} \mid\left\{\lambda q_{\mu}\right\}\right),
$$

where $D\left(\left\{f_{\mu}\right\} \mid\left\{\lambda_{\mu}\right\}\right)$ is a Dirichlet distribution [48, 49],

$$
D\left(\left\{f_{\mu}\right\} \mid\left\{\lambda_{\mu}\right\}\right) \equiv \frac{\Gamma(\lambda)}{\prod_{\mu^{\prime}} \Gamma\left(\lambda_{\mu^{\prime}}\right)} \prod_{\mu} f_{\mu}^{\lambda_{\mu}-1} .
$$

Here, $\lambda=\sum_{\mu=1}^{L} \lambda_{\mu}$. The (ensemble) average fraction reads $\left\langle f_{\mu}\right\rangle=\left(q_{\mu} \lambda+p_{\mu}\right) /(\lambda+1)$. When $p_{\nu}=q_{\nu}$, due to the normalization $\sum_{\nu=1}^{L} f_{\nu}=1$, the first factor in Eq. (17) does not contribute, and $\left\langle f_{\mu}\right\rangle=q_{\mu}$.

We notice that $\mathcal{P}\left(\left\{f_{\mu}\right\}\right)$ [Eq. (17)] depends on the initial conditions $\left\{p_{\mu}\right\}_{\mu=1}^{L}$. This property arises from the strong memory effects that drive the underlying stochastic dynamics. Nevertheless, this dependence is not able to cancel any of the stationary fractions. In consequence, the initial conditions are not relevant for breaking or not ergodicity. In fact, given that $\mathcal{P}\left(\left\{f_{\mu}\right\}\right)$ departs from Eq. (10), this model leads to EB. The distribution $P(\mathcal{O})[$ Eq. (9)] can be evaluated from Eq. (17).

As an example, we consider a two-level system, where the observable is defined by $\left\{\mathcal{O}_{\mu}\right\} \rightarrow\left(\mathcal{O}_{2}, \mathcal{O}_{1}\right)$, with $\mathcal{O}_{1} \leq$ $\mathcal{O} \leq \mathcal{O}_{2}$. After integration, we get

$$
P(\mathcal{O})=\frac{1}{\mathcal{N}} \frac{\left[\omega_{2}\left(\mathcal{O}_{2}-\mathcal{O}\right)\right]^{\lambda_{1}-1}\left[\omega_{1}\left(\mathcal{O}-\mathcal{O}_{1}\right)\right]^{\lambda_{2}-1}}{\left[\omega_{2}\left(\mathcal{O}_{2}-\mathcal{O}\right)+\omega_{1}\left(\mathcal{O}-\mathcal{O}_{1}\right)\right]^{\lambda_{1}+\lambda_{2}}},
$$

where for shortening the expression we introduced the parameters $\lambda_{1} \equiv \lambda q_{1}, \lambda_{2} \equiv \lambda q_{2}$, and the weights

$$
\omega_{1} \equiv \frac{\tau_{1}}{\tau_{1}+\tau_{2}}, \quad \omega_{2} \equiv \frac{\tau_{2}}{\tau_{1}+\tau_{2}} .
$$

Here, $\tau_{1}$ and $\tau_{2}$ are the average times corresponding to the two waiting time distributions $w_{1}(t)$ and $w_{2}(t)$ respectively [Eq. (10]. The normalization constant reads $\mathcal{N}^{-1}=\left(\mathcal{O}_{2}-\mathcal{O}_{1}\right) \omega_{1} \omega_{2} \Gamma\left(\alpha_{1}+\alpha_{2}\right) / \Gamma\left(\alpha_{1}\right) \Gamma\left(\alpha_{2}\right)$. For simplicity, in the previous expressions we assumed the initial condition $p_{\mu}=q_{u}$. The case $p_{\mu} \neq q_{u}$ can be recovered from these expressions [see Eqs. (17) and (18)].

The model (16) demonstrates that global memory effects may lead to EB. This result has a close relation with the breakdown of the standard central limit theorem for globally correlated random variables [50]. On the other hand, as shown in Sec. IV, depending on the values of $\lambda$, here $\mathrm{EB}$ arises because the residence times in each state may be divergent, that is, their probability density is characterized by power-law tails. The next modified dynamics also develops EB, but does not involve power-law statistics.

\section{Imperfect urn-like Model}

Here, we consider a model that can be seen as an imperfect case of the previous one. We consider the possibility of having random state selections that do not depend on the previous system history. The transition probability reads

$$
\mathcal{T}_{n}\left(\left\{n_{\nu}\right\} \mid \mu\right)=\varepsilon q_{\mu}+(1-\varepsilon) \frac{\lambda q_{\mu}+M_{\mu}}{M+\lambda} .
$$

The set $\left\{q_{\mu}\right\}_{\mu=1}^{L}$ is normalized as before, $0 \leq \varepsilon \leq 1$, and $\lambda \geq 0$. Hence, with probability $\varepsilon$ each state $\mu$, independently of the previous trajectory, is chosen with weight $q_{\mu}$. Complementarily, with probability $(1-\varepsilon)$ the state is chosen in agreement with the urn mechanism Eq. (16). In fact, here $M_{\mu}$ is the number of times that the state $\mu$ was chosen with the urn dynamics. Furthermore, $M$ is the number of times that the urn mechanism was applied, $M=\sum_{\mu=1}^{L} M_{\mu}$. In contrast with the elephant model [Eq. (15)], here the contribution proportional to $\varepsilon$ can be think as an error in the application of the urn dynamics.

In order to clarify the stochastic dynamics induced by Eq. (21), in Fig. 1 we plot two realizations (upper panels) for a two-level system with $\mathcal{O}_{2}=1$ and $\mathcal{O}_{1}=-1$. Hence, the observable realizations switch between these two values. The waiting time distributions are exponential ones

$$
w_{\mu}(t)=\gamma_{\mu} \exp \left[-\gamma_{\mu} t\right],
$$

with $\mu=1,2$. In the lower panels we plotted the conditional probability $\mathcal{T}_{n}\left(\left\{n_{\nu}\right\} \mid \mu\right)$ as a function of $n$. For clarity, each value is continued in the real interval $(i-1, i)$. The left panels corresponds to $\varepsilon=0.1$, Eq. (21), while the right panels to $\varepsilon=0$, that is, Eq. (16). In both cases $\mathcal{T}_{n}\left(\left\{n_{\nu}\right\} \mid \mu\right)$ attains stationary values for increasing $n$ [Eq. (2)]. Nevertheless, in the case $\varepsilon=0.1$ at random values of $n$ the conditional probability collapses to the value $q_{\mu}$. 

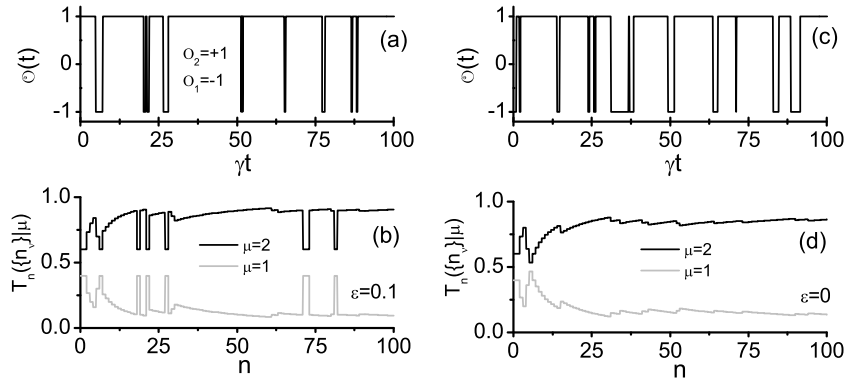

FIG. 1: Realizations of a two-level systems (upper panels) with observable $\left\{\mathcal{O}_{2}=1, \mathcal{O}_{1}=-1\right\}$ driven by an urn-like dynamics, jointly with the corresponding conditional probabilities $\mathcal{T}_{n}\left(\left\{n_{\nu}\right\} \mid \mu\right)$ [Eqs. (16) and (21)] as a function of $n$ (lower panels). The parameters are $\lambda=2, p_{1}=q_{1}=0.4$, and $p_{2}=q_{2}=0.6$. The waiting time distributions are exponential functions [Eq. (22)] with $\gamma_{1}=\gamma_{2}=\gamma$. In (a) and (b) $\varepsilon=0.1$, while in (c) and (d) we take $\varepsilon=0$.

This effect gives the error or imperfection with respect to the case $\varepsilon=0$.

The probability distribution of the asymptotic fractions [Eq. (2)] associated to Eq. (21) is given by

$$
\begin{aligned}
\mathcal{P}\left(\left\{f_{\mu}\right\}\right)= & \left\{\sum_{\nu=1}^{L} \frac{p_{\nu}}{q_{\nu}} \frac{f_{\nu}-\varepsilon q_{\nu}}{1-\varepsilon}\right\} \frac{1}{(1-\varepsilon)^{L-1}} \\
& D\left(\left\{\frac{f_{\mu}-\varepsilon q_{\mu}}{1-\varepsilon}\right\} \mid\left\{\lambda q_{\mu}\right\}\right),
\end{aligned}
$$

where $D\left(\left\{f_{\mu}\right\} \mid\left\{\lambda_{\mu}\right\}\right)$ is the Dirichlet distribution Eq. (18). Furthermore, each fraction is restricted to the domain

$$
\varepsilon q_{\mu} \leq f_{\mu} \leq 1-\varepsilon\left(1-q_{\mu}\right) .
$$

In this case, the average fraction reads

$$
\left\langle f_{\mu}\right\rangle=\frac{q_{\mu}(\lambda+\varepsilon)+p_{\mu}(1-\varepsilon)}{(\lambda+1)} .
$$

Eq. (23) is related to Eq. (17) by the change of variables $f_{\mu} \rightarrow \varepsilon q_{\mu}+(1-\varepsilon) f_{\mu}$. This relation follows by considering the asymptotic limits of Eqs. (21) and (16), and by using that the law of large numbers applies to the error mechanism. For $\varepsilon=0$ the previous expressions recover the previous case, Eq. (17). Interestingly, the effect of introducing the imperfect mechanism is to reduce the domain of each fraction $f_{\mu}$, Eq. (24).

From Eqs. (9) and (23) we can calculate the distribution of the time-averaged observable. Below we consider a two-level system with $\mathcal{O}_{1}<\mathcal{O}<\mathcal{O}_{2}$ and initial condition $p_{\mu}=q_{u}$. This case straightforwardly allows us to reconstruct the case $p_{\mu} \neq q_{u}$. We get

$$
\begin{aligned}
P(\mathcal{O})= & \frac{1}{\mathcal{N}_{\varepsilon}}\left[\omega_{2}\left(\mathcal{O}_{2}-\mathcal{O}\right)-\omega_{1}^{\varepsilon}\left(\mathcal{O}-\mathcal{O}_{1}\right)\right]^{\lambda_{1}-1} \\
& \times\left[\omega_{1}\left(\mathcal{O}-\mathcal{O}_{1}\right)-\omega_{2}^{\varepsilon}\left(\mathcal{O}_{2}-\mathcal{O}\right)\right]^{\lambda_{2}-1} \\
& \times \frac{1}{\left[\omega_{2}\left(\mathcal{O}_{2}-\mathcal{O}\right)+\omega_{1}\left(\mathcal{O}-\mathcal{O}_{1}\right)\right]^{\lambda_{1}+\lambda_{2}}} .
\end{aligned}
$$
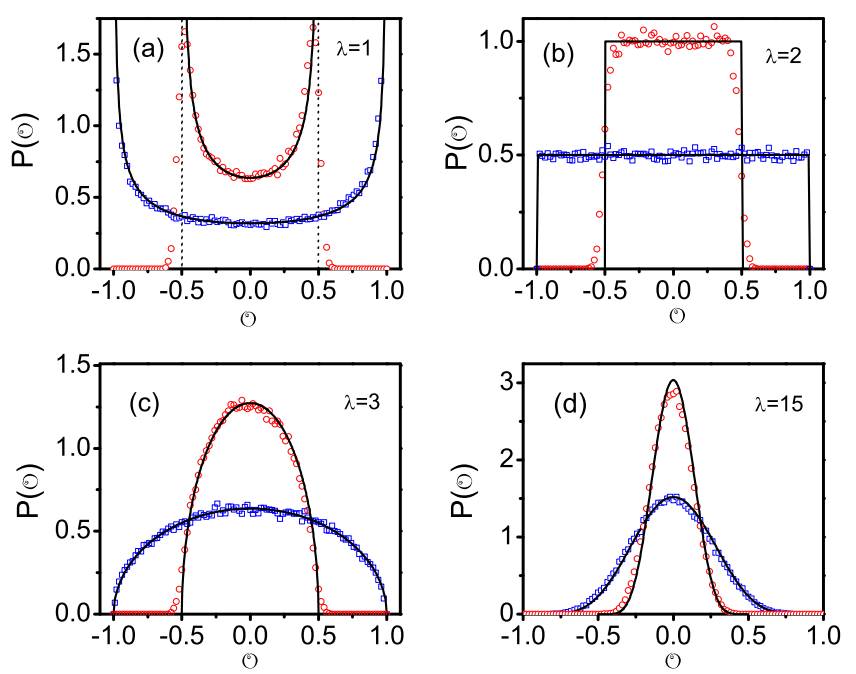

FIG. 2: Probability density of the time-averaged observable $\mathcal{O}$. We take a two-level system driven by an urn-like dynamics with different values of $\lambda$ and $\varepsilon$. The full lines correspond to the analytical expressions Eqs. (19) and (26). The waiting time distributions are exponential functions [Eq. (22)] with $\gamma_{1}=\gamma_{2}=\gamma$. In all plots we take $p_{1}=p_{2}=q_{1}=q_{2}=$ $1 / 2$. The (red) circles $(\varepsilon=0.5)$ and (blue) squares $(\varepsilon=0)$ correspond to numerical simulations. $\lambda$ is indicated in each plot.

The possible values of the time-averaged observable is restricted to the domain $\mathcal{O}_{\min } \leq \mathcal{O} \leq \mathcal{O}_{\max }$, where

$$
\mathcal{O}_{\max } \equiv \frac{\mathcal{O}_{2}+\mathcal{O}_{1} \omega_{1}^{\varepsilon} \omega_{2}^{-1}}{1+\omega_{1}^{\varepsilon} \omega_{2}^{-1}}, \quad \mathcal{O}_{\min } \equiv \frac{\mathcal{O}_{1}+\mathcal{O}_{2} \omega_{2}^{\varepsilon} \omega_{1}^{-1}}{1+\omega_{2}^{\varepsilon} \omega_{1}^{-1}}
$$

Furthermore, we introduced the parameters

$$
\omega_{1}^{\varepsilon} \equiv \omega_{1} \frac{\varepsilon q_{1}}{1-\varepsilon q_{1}}, \quad \omega_{2}^{\varepsilon} \equiv \omega_{2} \frac{\varepsilon q_{2}}{1-\varepsilon q_{2}},
$$

while the normalization constant is $\mathcal{N}_{\varepsilon}^{-1}=\left(\mathcal{O}_{2}-\right.$ $\left.\mathcal{O}_{1}\right) \omega_{1} \omega_{2}\left[\Gamma(\lambda) / \Gamma\left(\lambda_{1}\right) \Gamma\left(\lambda_{2}\right)\right](1-\varepsilon)^{-(\lambda-1)}\left(1-\varepsilon q_{1}\right)^{\lambda_{1}-1}(1-$ $\left.\varepsilon q_{2}\right)^{\lambda_{2}-1}$. Consistently, for $\varepsilon=0$, Eq. (26) recovers the previous case, Eq. (19). From the previous expression it become clear that the error mechanism introduced in Eq. (21) lead to a shrinking of the probability density of the time-averaged observable.

In order to check these results, in Fig. 2, we plot the distribution (26) for a two-level system where as before we take $\mathcal{O}_{2}=1, \mathcal{O}_{1}=-1$, and the exponential waiting time distributions (22). For each value of $\lambda$, we plot the cases $\varepsilon=0.5$ [Eqs. (21) and (26)] and $\varepsilon=0$ [Eqs. (16) and (19)]. Consistently, a higher $\varepsilon$ leads to a shrinking of the density $P(\mathcal{O})$, which confirms that for $\varepsilon \rightarrow 1$ an ergodic regime is achieved, $P(\mathcal{O})=\delta(\mathcal{O})$. The same happen for increasing $\lambda$. On the other hand, the plots show that $P(\mathcal{O})$ may develops different forms such as $U$ and bell shapes, or even uniform ones. Similar dependences arise when studying renewal random walks with divergent average trapping times [4]. 
In all cases, the numerical simulations (circles and squares) follows from a time average performed on a time interval with $n=10^{3}$ steps and $10^{5}$ realizations. The theoretical results fit very well the numerical ones.

\section{PROBABILITY DENSITY OF RESIDENCE TIMES}

In contrast to the elephant random walk model, the previous urn models develop weak EB. Here, we explore if this property is induced, or not, by a power-law statistics. In fact, for continuous-time random walks with renewal events, EB is induced by the divergence of the average residence time in each state [4]. The residence times are the random times that the system stays or remains in a given state before jumping to another one (see Fig. 1). Here, for the models introduced previously, we calculate their probability density. The calculations are valid for arbitrary number of states $L$.

We consider a single trajectory in the long time limit, such that the fractions $\left\{f_{\mu}\right\}_{\mu=1}^{L}$ [Eq. (2)] can be described by their associated probability density $\mathcal{P}\left(\left\{f_{\mu}\right\}\right)$ [see Eqs. (17) and (23)]. At the beginning of the residence in a given state $\mu$ the first time interval is chosen in agreement with its waiting time distribution $w_{\mu}(t)$. In each step, the system remains in the same state with probability $f_{\mu}$, which add a new random time interval also defined from $w_{\mu}(t)$. The residence time ends when a different state $\nu \neq \mu$ is chosen. This change occurs with probability $\left(1-f_{\mu}\right)$. Therefore, the probability $W_{\mu}(\{f\} \mid \tau) d \tau$ of leaving the state $\mu$ after a residence time $\tau$ can be written in the Laplace domain $\left[g(s)=\int_{0}^{\infty} d \tau g(\tau) e^{-s \tau}\right]$ as

$$
W_{\mu}(\{f\} \mid s)=\left(1-f_{\mu}\right) w_{\mu}(s) \sum_{n=0}^{\infty} f_{\mu}^{n} w_{\mu}^{n}(s) .
$$

Here, $w_{\mu}(s)$ is the Laplace transform of the waiting time distribution $w_{\mu}(t)$ associated to the state $\mu$. The previous expression takes into account all possible way of leaving the state $\mu$ after a given number of steps. It can be rewritten as

$$
W_{\mu}(\{f\} \mid s)=\left(1-f_{\mu}\right) \frac{w_{\mu}(s)}{1-f_{\mu} w_{\mu}(s)} .
$$

The density $W_{\mu}(\{f\} \mid \tau)$ is a conditional object. In fact, it is defined for a particular realization with random values of the fraction $f_{\mu}$. Therefore, the probability density of the residence time $W_{\mu}(t)$ is obtained after averaging over realizations, $W_{\mu}(t)=\left\langle W_{\mu}(\{f\} \mid t)\right\rangle$, which is equivalent to an average over the distribution $\mathcal{P}\left(\left\{f_{\nu}\right\}\right)$ of the set of fractions $\left\{f_{\nu}\right\}_{\nu=1}^{L}$. Therefore, we get

$$
W_{\mu}(\tau)=\int_{\Lambda} d f_{1} \cdots d f_{L-1} \mathcal{P}\left(\left\{f_{\nu}\right\}\right) W_{\mu}(\{f\} \mid \tau),
$$

where $W_{\mu}(\{f\} \mid \tau)$ follows from Eq. (30) after Laplace inversion. The average persistence time $T_{\mu}$ is defined by

$$
T_{\mu} \equiv \int_{0}^{\infty} d \tau \tau W_{\mu}(\tau) .
$$

The previous two expressions can be evaluated for arbitrary waiting time distributions and memory models. For an exponential waiting time distribution $w_{\mu}(t)=$ $\gamma_{\mu} \exp \left[-\gamma_{\mu} t\right]$ [Eq. (22)] with mean value $\tau_{\mu}=1 / \gamma_{\mu}$ [Eq. (1)], it follows $w_{\mu}(s)=\gamma_{\mu} /\left(s+\gamma_{\mu}\right)$. From Eq. (30), we get $W_{\mu}(\{f\} \mid s)=\left(1-f_{\mu}\right) \gamma_{\mu} /\left[s+\left(1-f_{\mu}\right) \gamma_{\mu}\right]$, which can be inverted as

$$
W_{\mu}(\{f\} \mid \tau)=\left(1-f_{\mu}\right) \gamma_{\mu} \exp \left[-\left(1-f_{\mu}\right) \gamma_{\mu} \tau\right] .
$$

For an ergodic system, characterized by the probability density $\mathcal{P}\left(\left\{f_{\nu}\right\}\right)$ given by Eq. (10), from Eq. (31) we get

$$
W_{\mu}(\tau)=\left(1-\left\langle f_{\mu}\right\rangle\right) \gamma_{\mu} \exp \left[-\left(1-\left\langle f_{\mu}\right\rangle\right) \gamma_{\mu} \tau\right] .
$$

This result is consistent with the definition of the underlying stochastic process that in each step allows the persistence in the same state. In fact, the average persistence time is $T_{\mu}=1 /\left[\gamma_{\mu}\left(1-\left\langle f_{\mu}\right\rangle\right)\right]$, indicating an increasing of the average persistence time with an increasing of the weight $\left\langle f_{\mu}\right\rangle$. On the other hand, in the localized regime [Eq. [13)], due to the absence of transitions, it is not possible to define $W_{\mu}(\tau)$.

Taking exponential waiting time distributions Eq. (22), for a two-level system $[\mu=1,2]$ characterized by the conditional probability (21) (imperfect urn model), after a simple change of variables, Eqs. (23) and (31) deliver

$$
W_{\mu}^{\varepsilon}(\tau)=\frac{1}{\mathcal{N}} \int_{0}^{1} d f \varphi_{\varepsilon} \exp \left[-\varphi_{\varepsilon} \tau\right] c_{\mu} f^{\lambda_{\mu}-1}(1-f)^{\lambda_{\mu^{\prime}}-1},
$$

where the super-index denotes the dependence on the parameter $\varepsilon . \lambda_{\mu}=\lambda q_{\mu}[\mu=1,2]$ while $\lambda_{\mu^{\prime}}\left[\mu^{\prime}=2,1\right]$ corresponds to the other system state, $\lambda_{\mu^{\prime}}=\lambda q_{\mu^{\prime}}=\lambda\left(1-q_{\mu}\right)$. The initial conditions appears through the contribution

$$
c_{\mu} \equiv \frac{p_{\mu}}{q_{\mu}} f+\frac{1-p_{\mu}}{1-q_{\mu}}(1-f) .
$$

The decay rate $\varphi_{\varepsilon}$ is

$$
\varphi_{\varepsilon} \equiv \gamma_{\mu}\left[1-\varepsilon q_{\mu}-(1-\varepsilon) f\right],
$$

while the normalization constant reads $\mathcal{N}^{-1}=\Gamma\left(\lambda_{1}+\right.$ $\left.\lambda_{2}\right) / \Gamma\left(\lambda_{1}\right) \Gamma\left(\lambda_{2}\right)$. Straightforwardly, the average persistence time, $T_{\mu}^{\varepsilon}=\int_{0}^{\infty} d \tau \tau W_{\varepsilon}(\tau)$, from Eq. (35) can then be written as

$$
T_{\mu}^{\varepsilon}=\frac{1}{\mathcal{N}} \int_{0}^{1} d f \frac{1}{\varphi_{\varepsilon}} c_{\mu} f^{\lambda_{\mu}-1}(1-f)^{\lambda_{\mu^{\prime}}-1} .
$$

Consistently, for $\varepsilon=1$ Eq. (35) recovers Eq. (34) with $\left\langle f_{\mu}\right\rangle=q_{\mu}$ [Eq. [25)]. Hence, $\gamma_{\mu} T_{\mu}^{1}=1 /\left(1-q_{\mu}\right)$. The same results arise when $\lambda \rightarrow \infty$. For arbitrary $\varepsilon$ and $\lambda$, 
from Eqs. (35) and (38) explicit analytical expressions can be found for both $W_{\mu}^{\varepsilon}(\tau)$ and $T_{\mu}^{\varepsilon}$ [see Appendix $\mathrm{D}$.

Interestingly, for $0<\varepsilon \leq 1$ (and any initial condition) the average residence time $T_{\mu}^{\varepsilon}$ is finite [see Eq. (D4)]. This is the main result of this section. In fact, this result demonstrates that weak EB may arise even in the absence of power-law statistical distributions with divergent average residence times. On the other hand, for the case $\varepsilon=0$, that is, the dynamics defined by the conditional probabilities (16), the average residence time $T_{\mu}^{0}$, depending on the parameter values, may be finite or infinite. From Eqs. (38) and Eq. (D4) we get

$$
\gamma_{\mu} T_{\mu}^{0}=\frac{\lambda-\left(\frac{1-p_{\mu}}{1-q_{\mu}}\right)}{\lambda\left(1-q_{\mu}\right)-1}, \quad \lambda>\frac{1}{\left(1-q_{\mu}\right)}>1 .
$$

Consistently, for increasing $\lambda$ this expression recovers the ergodic case [Eq. (34)], $\lim _{\lambda \rightarrow \infty} \gamma_{\mu} T_{\mu}^{0}=1 /\left(1-q_{\mu}\right)$. In the complementary region of possible values of $\lambda$, the average residence time is divergent,

$$
\gamma_{\mu} T_{\mu}^{0}=\infty, \quad \lambda \leq \frac{1}{\left(1-q_{\mu}\right)} .
$$

This last regime indicates that the density $W_{\mu}^{\varepsilon}(\tau)$ develops power-law tails. In fact, for long residence times, $\gamma_{\mu} \tau \gg 1$, from Eqs. (35) and (D1) it can be approximated as

$$
W_{\mu}^{0}(\tau) \approx \gamma_{\mu} C_{\mu}^{0}\left(\frac{1}{\gamma_{\mu} \tau}\right)^{\lambda\left(1-q_{\mu}\right)+1}
$$

which defines the previous finite and infinite average time regimes. The dimensionless constant reads $C_{\mu}^{0}=\left(p_{\mu} / q_{\mu}\right)\left(1-q_{\mu}\right) \Gamma(1+\lambda) / \Gamma\left(q_{\mu} \lambda\right)$. When $p_{\mu}=0$ $\left(p_{\mu^{\prime}}=1\right)$ the asymptotic behavior becomes $W_{\mu}^{0}(\tau) \approx$ $\left(1 / \gamma_{\mu} \tau\right)^{\lambda\left(1-q_{\mu}\right)+2}$, while for $W_{\mu^{\prime}}^{0}(\tau)$ is given by Eq. (41). We remark that in general $W_{\mu}^{\varepsilon}(\tau)(\varepsilon>0)$ may also develop power-law behaviors. Nevertheless, a multiplicative exponential factor always leads to finite averages times [see for example Eq. (42) below].

For particular values of the characteristic parameters, the integral results Eqs. (35) and (38) lead to simple expressions. Taking $p_{1}=q_{1}=1 / 2, p_{2}=q_{2}=1 / 2$, and $\lambda=2[$ Fig. (2b)] the density of residence times becomes

$$
W_{\mu}^{\varepsilon}(\tau)=\frac{\exp \left(-\gamma_{\varepsilon}^{+} \tau\right)\left(1+\gamma_{\varepsilon}^{+} \tau\right)-\exp \left(-\gamma_{\varepsilon}^{-} \tau\right)\left(1+\gamma_{\varepsilon}^{-} \tau\right)}{\gamma_{\mu} \tau^{2}(1-\varepsilon)},
$$

where for shortening the expression we introduced the rates $\gamma_{\varepsilon}^{+} \equiv \gamma_{\mu} \varepsilon / 2$ and $\gamma_{\varepsilon}^{-} \equiv \gamma_{\mu}(1-\varepsilon / 2)$. In the case $\varepsilon=1$ (ergodic dynamics), we get $W_{\mu}^{\varepsilon}(\tau)=$ $\left(\gamma_{\mu} / 2\right) T_{\mu}^{1} \exp \left[-\left(\gamma_{\mu} / 2\right) \tau\right]$. Hence, $T_{\mu}^{1}=2 / \gamma_{\mu}$. In the case $\varepsilon=0$ it reduces to

$$
W_{\mu}^{0}(\tau)=\frac{1}{\gamma_{\mu} \tau^{2}}\left[1-\left(1+\gamma_{\mu} \tau\right) \exp \left(-\gamma_{\mu} \tau\right)\right]
$$

which explicitly shows the presence of dominant powerlaw tails. The average residence time $[\mathrm{Eq}$. (38)], for
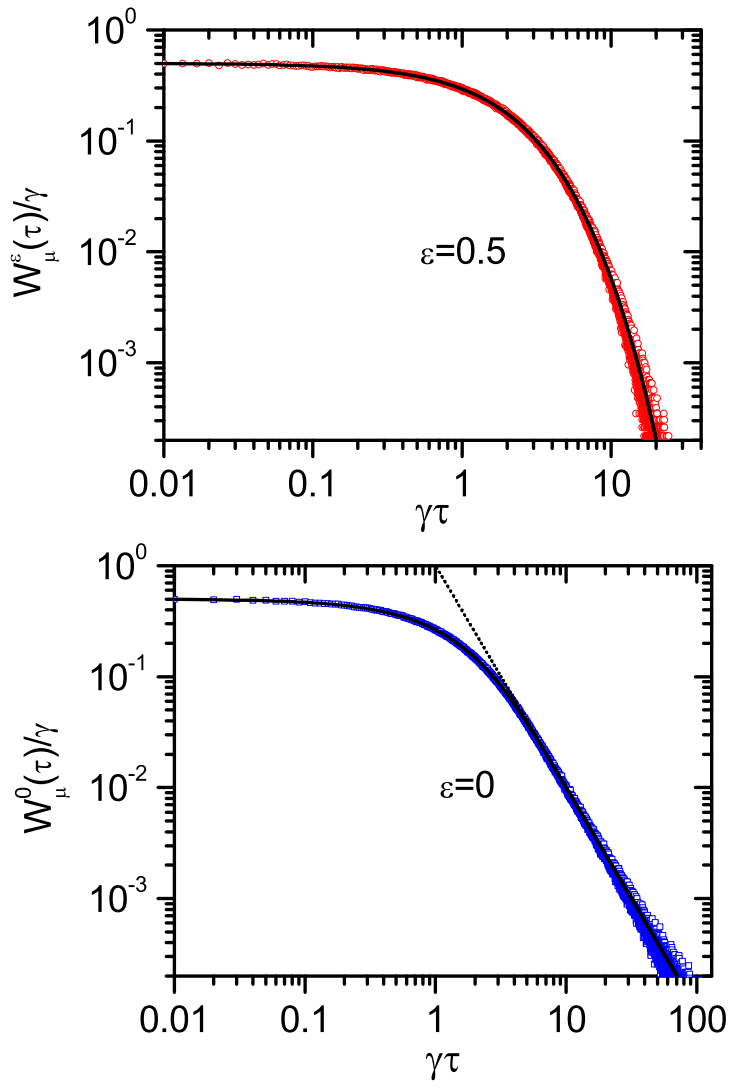

FIG. 3: Probability distribution $W_{\mu}^{\varepsilon}(\tau)[\mu=1,2]$ of the residence times for a two-level system. The full lines correspond to the analytical result Eq. (42). The waiting time distributions are exponential functions [Eq. (22)] with $\gamma_{1}=\gamma_{2}=\gamma$. In both curves, $p_{1}=p_{2}=q_{1}=q_{2}=1 / 2$, and $\lambda=2$. The (red) circles correspond to a numerical simulation with $\varepsilon=0.5$, while the (blue) squares to $\varepsilon=0$. The dotted line is the asymptotic power-law behavior (41) of Eq. (43).

arbitrary $\varepsilon$ reads

$$
\gamma_{\mu} T_{\mu}^{\varepsilon}=\frac{2 \operatorname{arctanh}(1-\varepsilon)}{(1-\varepsilon)}=\frac{\ln \left(\frac{2-\varepsilon}{\varepsilon}\right)}{(1-\varepsilon)},
$$

where $\operatorname{arctanh}[x]=\ln \sqrt{\frac{1+x}{1-x}}$ for $x \in(-1,1)$. Thus, $T_{\mu}^{\varepsilon}$ is finite for $0<\varepsilon \leq 1$. Consistently with Eqs. (40) and (43), it diverges for $\varepsilon=0, T_{\mu}^{0}=\lim _{\varepsilon \rightarrow 0} T_{\mu}^{\varepsilon}=\infty$.

In order to check the previous results we determined the distribution $W_{\mu}^{\varepsilon}(\tau)$ from a set of realizations such as those shown in Fig. 1. For the same system than in Fig. 2, the results are shown in Fig. 3. Furthermore, we take $w_{1}(t)=w_{2}(t)=\gamma \exp (-\gamma t)$, which implies $W_{1}^{\varepsilon}(\tau)=W_{2}^{\varepsilon}(\tau)$. Consistently with the previous analytical results [Eq. (42)], for $\varepsilon=0.5$ [Fig. 3(a)] asymptotically the density of residence times $W_{\mu}^{\varepsilon}(\tau)$ is not dominated by power-law behaviors. Instead for $\varepsilon=0$ [Fig. 3(b)] an asymptotic power-law behavior is clearly observed [Eq. (43)]. The numerical and theoretical results are consistent between them.

The numerical probability densities of Fig. 3 were ob- 
tained from a set of equally sampled realizations. This means that the same number of data for the random residence times are taken from each realization. We took $5 \times 10^{3}$ realizations with a total length of $n=5 \times 10^{5}$ steps. Furthermore, after running the dynamics during $10^{3}$ steps (long time limit), $5 \times 10^{3}$ random residence times were taken from each realization.

\section{SUMMARY AND CONCLUSIONS}

We have introduced a random walk dynamics characterized by global memory mechanisms. Given a finite set of states, in each step the system may remain in the same state of jump to another one. These alternative events are chosen from a conditional probability that depends on the whole previous history of the system. The time between consecutive steps is determinate by a set of waiting time distributions, all of them characterized by a finite average time.

We focused the analysis on the ergodic properties of the stochastic dynamics. Hence, we characterized the probability density of time-averaged observables, [Eq. (9)]. By analyzing different memory mechanisms, we conclude that global correlations are not a sufficient condition for breaking ergodicity, such as for example in the elephant random walk model [Eq. (15)]. On the other hand, alternative urn-like memory mechanisms [Eqs. (16) and (21)] do in fact break ergodicity. In these cases, considering a two-level dynamics, the distribution of time-averaged observables can be found in an explicit analytical way [Eqs. (19) and (26)].

For random walks dynamics over a finite set of states, EB may be induced by a divergent average residence time in each state. In order to cheek this possibility for the present models, we calculated the probability density of the residence times [Eq. (31)], and the corresponding average residence time [Eq. (32)]. In general, the distributions do not develop asymptotic power-law behaviors consistent with a divergent average residence time. Hence, we conclude that global memory effects are in fact an alternative mechanism that leads to EB. This main conclusion was explicitly checked for two-level dynamics [Eqs. (35]) and (38)]. Only for a particular set of values, the residence times have a divergent average. All previous results were confirmed by numerical simulations [see Figs. (2) and (3)].

In conclusion, we established that weak EB may arise in systems characterized by global memory effects. This property may emerge even when the relevant variables are not characterized by power-law statistical behaviors.

\section{Acknowledgments}

This work was supported by Consejo Nacional de Investigaciones Científicas y Técnicas (CONICET), Argentina.

\section{Appendix A: Ensemble probabilities and stationary state}

Here, we obtain the ensemble probabilities $\left\{P_{\mu}(t)\right\}_{\mu=1}^{L}$ and their corresponding long time limit, Eq. (4).

From the dynamics defined in Sec. II, the probability $P_{\mu}(t)$ that the system is in the (arbitrary) state $\mu$ at time $t$, can be written in the Laplace domain $[g(s)=$ $\left.\int_{0}^{\infty} d \tau g(\tau) e^{-s \tau}\right]$ as

$$
\begin{aligned}
P_{\mu}(s)= & P_{1}(\mu) \Phi_{\mu}(s)+\sum_{n=1}^{\infty} \sum_{\mu_{1}, \cdots \mu_{n}} P_{n+1}\left(\mu_{1}, \cdots \mu_{n}, \mu\right) \\
& \times w_{\mu_{1}}(s) \cdots w_{\mu_{n}}(s) \Phi_{\mu}(s),
\end{aligned}
$$

where $\Phi_{\mu}(s)=\left[1-w_{\mu}(s)\right] / s$ is the Laplace transform of the survival probability $\Phi_{\mu}(t)=1-\int_{0}^{t} d t^{\prime} w_{\mu}\left(t^{\prime}\right)$. Furthermore, $P_{n}\left(\mu_{1}, \cdots, \mu_{n}\right)$ is the probability of obtaining, after $n$ steps, the states $\left\{\mu_{1}, \cdots, \mu_{n}\right\}$ from the globally correlated mechanism. Hence, $P_{1}(\mu)=p_{\mu}$.

Eq. (A1) can be seen an addition over the ensemble realizations, where each term gives the weight of all realizations with $n$-selection events. Taking into account that the variables $\mu_{1}, \cdots, \mu_{n-1}$ runs over the domain of possible states $1,2, \cdots L$, Eq. A1 can also be written as

$$
\begin{aligned}
P_{\mu}(s)= & p_{\mu} \Phi_{\mu}(s)+\sum_{n=1}^{\infty} \sum_{\left\{n_{\nu}\right\}} P_{n}\left(n_{1}, \cdots n_{L}\right) \\
& \times \mathcal{T}_{n}\left(\left\{n_{\nu}\right\} \mid \mu\right) w_{1}^{n_{1}}(s) \cdots w_{L}^{n_{L}}(s) \Phi_{\mu}(s) .
\end{aligned}
$$

Here, $P_{n}\left(n_{1}, \cdots n_{L}\right)$ is the joint probability of getting $n_{\nu}$ times the state $\nu$ after $n$-random steps, $\nu=1, \cdots L$. Therefore, the sum $\sum_{\left\{n_{\nu}\right\}}$ is restricted to the condition $\sum_{\nu=1}^{L} n_{\nu}=n$.

The expression (A2) is exact. Now, we perform a set of approximations for getting the stationary state $P_{\mu}^{\mathrm{st}}=$ $\lim _{t \rightarrow \infty} P_{\mu}(t)$. In the long time regime, $t \gg\left\{\tau_{\nu}\right\}_{\nu=1}^{L}$, in the Laplace domain we can approximate [46] the waiting time distribution as $w_{\nu}(s) \simeq 1-\tau_{\nu} s$, where $\tau_{\nu}$ is the average time defined by Eq. (11). Therefore, $\Phi_{\mu}(s) \simeq \tau_{\mu}$ and also $w_{1}^{n_{1}}(s) \cdots w_{L}^{n_{L}}(s)=\prod_{\nu=1}^{L} w_{\nu}^{n_{\nu}}(s) \simeq$ $\exp \left[-s \sum_{\nu=1}^{L} \tau_{\nu} n_{\nu}\right]$, which in the time domain leads to a Dirac delta function, $\delta\left(t-\sum_{\nu=1}^{L} \tau_{\nu} n_{\nu}\right)$.

In the long time regime, $n$ increases unbounded. For the studied models, the conditional probability can then be approximated as $\mathcal{T}_{n}\left(\left\{n_{\nu}\right\} \mid \mu\right) \simeq n_{\mu} / n \simeq f_{\mu}$ [Eq. (2)] . Consequently, Eq. (A2) leads to the approximation

$$
P_{\mu}(t) \simeq \sum_{n=1}^{\infty} \sum_{\left\{n_{\nu}\right\}} P_{n}\left(n_{1}, \cdots n_{L}\right) \tau_{\mu} \frac{n_{\mu}}{n} \delta\left(t-\sum_{\nu=1}^{L} \tau_{\nu} n_{\nu}\right)
$$

By writing the delta contribution as $\delta\left(t-\sum_{\nu=1}^{L} \tau_{\nu} n_{\nu}\right)=$ $\delta\left(t-n \sum_{\nu=1}^{L} \tau_{\nu} f_{\nu}\right)$, we realize that in the sum over $n$ the dominant term is that with $n \simeq t / \sum_{\nu=1}^{L} \tau_{\nu} f_{\nu}$. Using the properties of the delta distribution, $\delta(t-$ 
$\left.n \sum_{\nu=1}^{L} \tau_{\nu} f_{\nu}\right)=\left(1 / \sum_{\nu=1}^{L} \tau_{\nu^{\prime}} f_{\nu^{\prime}}\right) \delta\left(n-t / \sum_{\nu=1}^{L} \tau_{\nu} f_{\nu}\right)$, and after the change of variables $n_{\nu} \rightarrow f_{\nu}$, Eq. (A3) leads to the stationary state

$$
P_{\mu}^{\mathrm{st}}=\int_{\Lambda} d f_{1} \cdots d f_{L-1} \frac{\tau_{\mu} f_{\mu}}{\sum_{\nu=1}^{L} \tau_{\nu} f_{\nu}} \mathcal{P}\left(\left\{f_{\nu}\right\}\right),
$$

which in fact recovers Eq. (44). This result was also checked by numerical calculations for the memory models introduced in Sec. III.

\section{Appendix B: Ergodicity of the elephant random walk}

The elephant random walk is defined by the transition probability (15),

$$
\mathcal{T}_{n}\left(\left\{n_{\nu}\right\} \mid \mu\right)=\varepsilon q_{\mu}+(1-\varepsilon) \frac{n_{\mu}}{n} .
$$

Here, we demonstrate that the fractions defined in Eq. (2), $f_{\mu}=\lim _{n \rightarrow \infty}\left(n_{\mu} / n\right)$, converges to $q_{\mu}$, that is, the distribution of the fractions is given by Eq. (10) with $\left\langle f_{\mu}\right\rangle=q_{\mu}(0<\varepsilon \leq 1)$.

At a given stage, the numbers $n_{\mu}$ can be split as follows

$$
n_{\mu}=m_{\mu}^{(1)}+M_{\mu}^{(1)} .
$$

Here, $m_{\mu}^{(1)}$ gives the number of times that, with probability $\varepsilon$, the state $\mu$ was chosen with probabilities $\left\{q_{\mu}\right\}_{\mu=1}^{L}$. Complementarily, $M_{\mu}^{(1)}$ gives the number of times that, with probability $1-\varepsilon$, the state $\mu$ was chosen with probabilities $\left\{n_{\mu} / n\right\}$. In the limit of a diverging number of selections (steps), the law of large numbers gives $\lim _{n \rightarrow \infty} m_{\mu}^{(1)} / n=\varepsilon q_{\mu}$. Thus, asymptotically we can approximate

$$
\mathcal{T}_{n}\left(\left\{n_{\nu}\right\} \mid \mu\right) \simeq \varepsilon q_{\mu}+(1-\varepsilon)\left[\varepsilon q_{\mu}+\frac{M_{\mu}^{(1)}}{n}\right] .
$$

Now, we can split $M_{\mu}^{(1)}$ in the same way as follows

$$
M_{\mu}^{(1)}=m_{\mu}^{(2)}+M_{\mu}^{(2)} .
$$

Here, $m_{\mu}^{(2)}$ is the number of times that, with probability $(1-\varepsilon) \varepsilon$, the state $\mu$ was chosen with probabilities $\left\{q_{\mu}\right\}_{\mu=1}^{L}$. Similarly, $M_{\mu}^{(2)}$ gives the number of times that, with probability $(1-\varepsilon) \times(1-\varepsilon)$, the state $\mu$ was chosen with probabilities $\left\{M_{\mu}^{(1)} / n\right\}$. By using that $\lim _{n \rightarrow \infty} m_{\mu}^{(2)} / n=(1-\varepsilon) \varepsilon q_{\mu}$, it follows the approximation

$$
\mathcal{T}_{n}\left(\left\{n_{\nu}\right\} \mid \mu\right) \simeq \varepsilon q_{\mu}+(1-\varepsilon)\left[\varepsilon q_{\mu}+\varepsilon q_{\mu}(1-\varepsilon)+\frac{M_{\mu}^{(2)}}{n}\right] .
$$

Performing the same splitting, at an arbitrary order we can write

$$
M_{\mu}^{(k-1)}=m_{\mu}^{(k)}+M_{\mu}^{(k)}
$$

where the law of large numbers gives $\lim _{n \rightarrow \infty} m_{\mu}^{(k)} / n=$ $(1-\varepsilon)^{k-1} \varepsilon q_{\mu}$. Therefore, we get

$$
\mathcal{T}_{n}\left(\left\{n_{\nu}\right\} \mid \mu\right) \simeq \varepsilon q_{\mu}+(1-\varepsilon) \varepsilon q_{\mu} \sum_{k=0}^{\infty}(1-\varepsilon)^{k}=q_{\mu} .
$$

This argument shows that in the asymptotic limit the memory on the previous states is lost. Hence, the finite random walk becomes ergodic, Eq. (10) with $\left\langle f_{\mu}\right\rangle=q_{\mu}$. Numerical simulations confirm this result. Notice that the previous argument does not apply to the urn models Eqs. (16) and (21). On the other hand, we checked that for $\varepsilon \rightarrow 0$ the rate of convergence to the regime defined by Eq. (B7) is smaller, being infinite for $\varepsilon=0$, that is, in the localized regime. We remark that this result does not contradict previous results for unbounded diffusion processes [37 39].

\section{Appendix C: Fraction probability density of the urn-like dynamics}

For the urn dynamics defined by Eq. (16), here we obtain the probability density of the stationary fractions Eq. (2).

By using Bayes rule, the joint probability $P_{n}\left(\mu_{1}, \cdots \mu_{n}\right)$ of obtaining the values $\mu_{1}, \cdots \mu_{n}$ with the dynamics Eq. (16) can be written as

$P_{n}\left(\mu_{1}, \cdots \mu_{n}\right)=P_{1}\left(\mu_{1}\right) \mathcal{T}_{1}\left(\left\{n_{\nu_{1}}\right\} \mid \mu_{2}\right) \cdots \mathcal{T}_{n-1}\left(\left\{n_{\nu_{n-1}}\right\} \mid \mu_{n}\right)$.

By writing this expression in an explicit way, we realize that the joint probability $P_{n}\left(n_{1}, \cdots n_{L}\right)$ of getting $n_{\mu}$ times the state $\mu$ after $n$-random steps can be written as

$$
\begin{aligned}
P_{n}\left(n_{1}, \cdots n_{L}\right)= & \sum_{\nu=1}^{L} \frac{(n-1) !}{n_{1} ! \cdots\left(n_{\nu}-1\right) ! \cdots n_{L} !} \\
& \times p_{\nu} \frac{\Gamma(\lambda)}{\Gamma(n+\lambda)} \frac{1}{q_{\nu}} \prod_{\mu=1}^{L} \frac{\Gamma\left(n_{\mu}+\lambda_{\mu}\right)}{\Gamma\left(\lambda_{\mu}\right)},
\end{aligned}
$$

where $\lambda_{\mu}=\lambda q_{\mu}$. Each term in the sum $\sum_{\nu=1}^{L}$ corresponds to all realizations with the same initial condition, which leads to the weight $p_{\nu}$. The contributions proportional to the Gamma functions follows straightforwardly from the product of successive conditional probabilities $\mathcal{T}_{k}\left(\left\{n_{k}\right\} \mid \mu_{k+1}\right)$ and the property $\Gamma(n+x) / \Gamma(x)=$ $x(1+x)(2+x) \cdots(n-1+x)$. Furthermore, in the first line the multinomial factor takes into account all realizations with the same numbers $\left\{n_{\mu}\right\}_{\mu=1}^{L}$. Eq. (C1) can be rewritten as

$$
P_{n}\left(n_{1}, \cdots n_{L}\right)=\sum_{\nu=1}^{L} \frac{p_{\nu}}{q_{\nu}} \frac{n_{\nu}}{n} D_{n}\left(n_{1}, \cdots n_{L}\right),
$$

where

$$
D_{n}\left(n_{1}, \cdots n_{L}\right) \equiv \frac{n !}{n_{1} ! \cdots n_{L} !} \frac{\Gamma(\lambda)}{\Gamma(n+\lambda)} \prod_{\mu=1}^{L} \frac{\Gamma\left(n_{\mu}+\lambda_{\mu}\right)}{\Gamma\left(\lambda_{\mu}\right)} .
$$


In the limit $x \rightarrow \infty$ it is valid the Stirling approximation $\Gamma(x) \approx \sqrt{2 \pi / x} e^{-x} x^{x}$. Hence, in the same limit, it follows $\Gamma(x+\alpha) / \Gamma(x) \approx x^{\alpha}$. Using that $n !=\Gamma(n+1)$, and applying the previous approximations to Eq. (C3), in the limit $n \rightarrow \infty$ it follows

$$
D_{n}\left(n_{1}, \cdots n_{L}\right) \approx \frac{\Gamma(\lambda)}{n^{\lambda-1}} \prod_{\mu=1}^{L} \frac{n_{\mu}^{\lambda_{\mu}-1}}{\Gamma\left(\lambda_{\mu}\right)} .
$$

By performing the change of variables $n_{\mu} \rightarrow n f_{\mu}$, and by using that (due to normalization) there are $(L-1)$ independent variables $f_{\mu}$, the previous expression straightforwardly leads to the Dirichlet distribution $D\left(\left\{f_{\mu}\right\} \mid\left\{\lambda_{\mu}\right\}\right)$, Eq. (18). Therefore, in the same limit, Eq. (C2) trivially recovers Eq. (17).

\section{Appendix D: Exact analytical results for two-level systems}

For two-levels systems driven by the imperfect urn dynamics, the integrals expressions for the probability density of residence times [Eq. (35)] and the average residence time [Eq. (38)] can be explicitly evaluated. $W_{\mu}^{\varepsilon}(\tau)$ reads

$$
\begin{aligned}
W_{\mu}^{\varepsilon}(\tau)= & \gamma_{\mu} \exp \left[-\gamma_{\mu} \tau\left(1-\varepsilon q_{\mu}\right)\right] \\
& \left\{a_{\mu}(\tau){ }_{1} F_{1}\left[\lambda q_{\mu} ; \lambda ;(1-\varepsilon) \gamma_{\mu} \tau\right]\right. \\
& \left.+b_{\mu}(\tau)_{1} F_{1}\left[\lambda q_{\mu} ; \lambda+1 ;(1-\varepsilon) \gamma_{\mu} \tau\right]\right\}
\end{aligned}
$$

The Kummer confluent hypergeometric function is ${ }_{1} F_{1}[a ; b ; z]=\sum_{k=0}^{\infty}(a)_{k}(b)_{k} z^{k} / k !$ with $(x)_{k}=\prod_{j=0}^{k-1}(x+$ $j)=\Gamma(x+k) / \Gamma(x)$. The auxiliary function $a_{\mu}(\tau)$ is

$$
a_{\mu}(\tau) \equiv \frac{p_{\mu}}{q_{\mu}}\left(1-q_{\mu}\right) \varepsilon+\frac{\left(p_{\mu}-q_{\mu}\right) \lambda}{q_{\mu} \gamma_{\mu} \tau},
$$

while $b_{\mu}(\tau)$ is

$$
b_{\mu}(\tau) \equiv\left(1-\frac{p_{\mu}}{q_{\mu}} \varepsilon\right)-(1-\varepsilon) p_{\mu}+\left(p_{\mu}-q_{\mu}\right)\left(\varepsilon-\frac{\lambda}{q_{\mu} \gamma_{\mu} \tau}\right) .
$$

Similarly, the average residence time is

$$
\begin{aligned}
\gamma_{\mu} T_{\mu}^{\varepsilon}= & a_{\mu{ }_{2}} F_{1}\left[1 ; \lambda q_{\mu} ; \lambda ; \frac{1-\varepsilon}{1-\varepsilon q_{\mu}}\right] \\
& +b_{\mu}{ }_{2} F_{1}\left[1 ; 1+\lambda q_{\mu} ; 1+\lambda ; \frac{1-\varepsilon}{1-\varepsilon q_{\mu}}\right]
\end{aligned}
$$

Here, the hypergeometric function is defined by ${ }_{2} F_{1}[a ; b ; c ; z]=\sum_{k=0}^{\infty}(a)_{k}(b)_{k}(c)_{k} z^{k} / k$ !, while the coefficients are

$$
a_{\mu} \equiv \frac{1-p_{\mu}}{\left(1-q_{\mu}\right)\left(1-\varepsilon q_{\mu}\right)}, \quad b_{\mu} \equiv \frac{p_{\mu}-q_{\mu}}{\left(1-q_{\mu}\right)\left(1-\varepsilon q_{\mu}\right)}
$$

[1] N. Goldenfeld, Lectures on phase transitions and the renormalization group, (Perseus, 1992).

[2] J. P. Bouchaud, Weak ergodicity breaking and aging in disordered systems, J. Phys. I 2, 1705 (1992).

[3] E. Lutz, Power-Law Tail Distributions and Nonergodicity, Phys. Rev. Lett. 93, 190602 (2004).

[4] A. Rebenshtok and E. Barkai, Weakly Non-Ergodic Statistical Physics, J. Stat. Phys. 133, 565 (2008); A. Rebenshtok and E. Barkai, Distribution of Time-averaged Observables for Weak Ergodicity Breaking, Phys. Rev. Lett. 99, 210601 (2007).

[5] G. Margolin and E. Barkai, Nonergodisity of a time series obeying Lévy statistics, J. Stat. Phys. 122, 137 (2006).

[6] G. Bel and E. Barkai, Stochastic Ergodicity Breaking: a random Walk Approach, Phys. Rev. Lett. 94, 240602 (2005); G. Bel and E. Barkai, A Random Walk to a Non-Ergodic Equilibrium Concept, Phys. Rev. E 73, 016125 (2006); J. H. P. Schulz and E. Barkai, Fluctuations around equilibrium laws in ergodic continuous-time random walks, Phys. Rev. E 91, 062129 (2015).

[7] A. Saa and R. Venegeroles, Ergodic transitions in continuous-time random walks, Phys. Rev. E 82, 031110 (2010).

[8] T. Albers and G. Radons, Subdiffusive continuous time random walks and weak ergodicity breaking analyzed with the distribution of generalized diffusivities, Euro Phys. Lett. 102, 40006 (2013).
[9] M. Dentz, A. Russian, and P. Gouze, Self-averaging and ergodicity of subdiffusion in quenched random media, Phys. Rev. E 93, 010101(R) (2016.)

[10] A. G. Cherstvy, A. V. Chechkin, and R. Metzler, Anomalous diffusion and ergodicity breaking in heterogeneous diffusion processes, New J. of Phys. 15, 083039 (2013); A. G. Cherstvy and R. Metzler, Non-ergodicity, fluctuations, and criticality in heterogeneous diffusion processes, Phys. Rev. E 90, 012134 (2014).

[11] F. Kindermann, A. Dechant, M. Hohmann, T. Lausch, D. Mayer, F. Schmidt, E. Lutz, and A. Widera, Nonergodic Diffusion of Single Atoms in a Periodic Potential, arXiv:1601.0666 (2016); M. Khoury, A. M. Lacasta, J. M. Sancho, and K. Lindenberg, Weak disorder: anomalous transport and diffusion are normal yet again, Phys. Rev. Lett. 106, 090602 (2010).

[12] P. Massignan, C. Manzo, J. A. Torreno-Pina, M. F. García-Parajo, M. Lewestein, and G. J. Lapeyre, Jr., Nonergodic Subdiffusion from Brownian Motion in a Inhomogeneous Medium, Phys. Rev. Lett. 112, 150603 (2014).

[13] O. Peters, Ergodicity breaking in geometric Brownian motion, Phys. Rev. Lett. 110, 100603 (2013).

[14] H. Safdari, A. G. Cherstvy, A. V. Chechkin, F. Thiel, I. M. Sokolov, and R. Metzler, Quantifying the nonergodicity of scaled Brownian motion, J. Phys. A 48, 375002 (2015); H. Safdari, A. V. Chechkin, G. R. Jafari, 
and R. Metzler, Aging scaled Brownian motion, Phys. Rev. E 91, 042107 (2015).

[15] A. Godec, A. V. Chechkin, E. Barkai, H. Kantz, and R. Metzler, Localization and universal fluctuations in ultraslow diffusion processes, J. Phys. A 47, 492002 (2014); A. S. Bodrova, A. G. Cherstvy, A. V. Chechkin, and R. Metzler, Ultraslow scaled Brownian motion, arXiv:1503.08125 (2015).

[16] A. Godec and R. Metzler, Finite-Time Effects and Ultraweak Ergodicity Breaking in Superdiffusive Dynamics, Phys. Rev. Lett. 110, 020603 (2013).

[17] G. Bel and I. Nemenman, Ergodic and non-ergodic anomalous diffusion in coupled stochastic processes, New. J. Phys. 11, 083009 (2009).

[18] Y. Meroz, I. M. Sokolov and J. Klafter, Subdiffusion of mixed origins: When ergodicity and nonergodicity coexist, Phys. Rev. E 81, 010101(R) (2010); F. Thiel and I. M. Sokolov, Weak ergodicity breaking in an anomalous diffusion process of mixed origins, Phys. Rev. E 89, 012136 (2014).

[19] A. Fulinski, Anomalous diffusion and weak nonergodicity, Phys. Rev. E 83, 061140 (2011).

[20] A. Dechant, E. Lutz, D. A. Kessler, and E. Barkai, Fluctuations of Time Averages for Langevin Dynamics in a Binding Force Field, Phys. Rev. Lett. 107, 240603 (2011).

[21] G. Bel and E. Barkai, Ergodicity Breaking in a Deterministic Dynamical System, EuroPhys. Lett. 74, 15 (2006);

[22] T. Albers and Günter Radons, Weak Ergodicity Breaking and Aging of Chaotic Transport in Hamiltonian Systems, Phys. Rev. Lett. 113, 184101 (2014);

[23] A. Figueiredo, T. M. Rocha Filho, M. A. Amato, Z. T. Oliveira, Jr., and R. Matsushita, Truncated Lévy flights and weak ergodicity breaking in the Hamiltonian meanfield model, Phys. Rev. E 89, 022106 (2014).

[24] T. Akimoto, Distributional Response to Biases in Deterministic Superdiffusion, Phys. Rev. Lett. 108, 164101 (2012).

[25] X. Brokmann, J.-P. Hermier, G. Messin, P. Desbiolles, J.-P. Bouchaud, and M. Dahan, Statistical Aging and Nonergodicity in the Fluorescence of Single Nanocrystals, Phys. Rev. Lett. 90, 120601 (2003).

[26] G. Margolin and E. Barkai, Nonergodicity and Blinking Nanocrystals and Other Lévy-Walk processes, Phys. Rev. Lett. 94, 080601 (2005).

[27] C. Manzo, J. Arreno-Pina, P. Massignan, G. J. Lapeyre, Jr. M. Lewenstein, and M. F. Garcia Parajo, Weak Ergodicity Breaking of Receptor Motion in Living Cells Stemming from Random Diffusivity, Phys. Rev. X 5, 011021 (2015).

[28] Y. He, S. Burov, R. Metzler, and E. Barkai, Random Time-Scale Invariant Diffusion and Transport Coefficients, Phys. Rev. Lett. 101, 058101 (2008).

[29] A. Lubelski, I. M . Sokolov, and J. Klafter, Nonergodicity Mimics Inhomogeneity in Single Particle Tracking, Phys. Rev. Lett. 100, 250602 (2008).

[30] S. Burov, J. -H. Jeon, R. Metzler, and E. Barkai, Single particle tracking in systems showing anomalous diffusion: the role of weak ergodicity breaking, Phys. Chem. Chem. Phys. 13, 1800 (2011).

[31] J. Jeon, V. Tejedor, S. Burov, E. Barkai, C. SelhuberUnkel, K. Berg-Sorensen, L. Oddershede, and R. Metzler, In Vivo Anomalous Diffusion and Weak Ergodicity Breaking of Lipid Granules, Phys. Rev. Lett. 106, 048103
(2011).

[32] A. V. Weigel, B. Simon, M. M. Tamkun, and D. Krapf, Ergodic and nonergodic processes coexist in the plasma membrane as observed by single-molecule tracking, Proc. Natl. Acad. Sci. U.S.A. 108, 6438 (2011).

[33] B. J. West, E. L. Geneston, and P. Grigolini, Maximizing information exchange between complex networks, Phys. Rep. 468, 1 (2008); and references there in.

[34] N. Piccinini, D. Lambert, B. J. West, M. Bologna, and P. Grigolini, Non-ergodic Complexity Management, arXiv:1511.08140 (2016); E. Geneston, R. Tuladhar, M. T. Beig, M. Bologna, and P. Grigolini, Ergodicity Breaking and Localization, arXiv:1601.02879 (2015).

[35] L. Silvestri, L. Fronzoni, P. Grigolini, and P. Allegrini, Event-Driven Power-Law Relaxation in Weak Turbulence, Phys. Rev. Lett. 102, 014502 (2009).

[36] S. Bianco, M. Ignaccolo, M. S. Rider, M. J. Ross, P. Winsor, and P. Grigolini, Brain, Music and non-Poisson Renewal Processes, Phys. Rev. E 75, 061911 (2007).

[37] G. M. Schütz and S. Trimper, Elephants can always remember: Exact long-range memory effects in a nonMarkovian random walk, Phys. Rev. E 70, 045101(R) (2004).

[38] H. Kim, Anomalous diffusion induced by enhancement of memory, Phys. Rev. E 90, 012103 (2014).

[39] R. Kürsten, Random recursive trees and the elephant random walk, Phys. Rev. E 93, 032111 (2016).

[40] J. C. Cressoni, M. A. A. da Silva, and G. M. Viswanathan, Amnestically Induced Persistence in Random Walks, Phys. Rev. Lett. 98, 070603 (2007); A. S. Ferreira, J. C. Cressoni, G. M. Viswanathan, and M. A. Alves da Silva, Anomalous diffusion in nonMarkovian walks having amnestically induced persistence, Phys. Rev. E 81, 011125 (2010); J. C. Cressoni, G. M. Viswanathan, and M. A. A. da Silva, Exact solution of an anisotropic $2 \mathrm{D}$ random walk model with strong memory correlations, J. Phys. A 46, 505002 (2013).

[41] V. M. Kenkre, Analytic formulation, Exact Solutions, and Generalizations of the elephant and the Alzheimer Random Walks, arXiv:0708.0034 (2007).

[42] N. Kumar, U. Harbola, and K. Lindenberg, Memoryinduced anomalous dynamics: emergence of diffusion, subdiffusion, and superdiffusion from a single random walk model, Phys. Rev. E 82, 021101 (2010).

[43] D. Boyer and J. C. Romo-Cruz, Solvable random-walk model with memory and its relations with Markovian models of anomalous diffusion, Phys. Rev. E 90, 042136 (2014).

[44] F. N. C. Paraan and J. P. Esguerra, Exact moments in a continuous time random walk with complete memory of its history, Phys. Rev. E 74, 032101 (2006).

[45] R. Hanel and S. Thurner, Generalized (c,d)-Entropy and Aging Random Walks, Entropy 15, 5324 (2013).

[46] W. Feller, An introduction to probability theory and applications, Vol. I \& II, (John Wiley \& Sons, 1967).

[47] N. L. Johnson and S. Kotz, Urn Models and Their Application, (John Wiley\&Sons, 1977).

[48] J. Pitman, Combinatorial Stochastic Processes, (Springer 2006).

[49] D. Blackwell and J. B. MacQueen, Fergurson distributions via Pólya urn schemes, The Annals of Statistics 1, 353 (1973).

[50] A. A. Budini, Central limit theorem for a class of globally correlated random variables, arXiv:1603.07314. 